\title{
Stable transplantation results of magnetically retracted islets: a novel method
}

\author{
G. G. M. Pinkse ${ }^{1,2}$, E. Steenvoorde ${ }^{1}$, S. Hogendoorn 1 , M. Noteborn ${ }^{3}$, O. T. Terpstra ${ }^{2}$, J. A. Bruijn ${ }^{1}$, E. de Heer ${ }^{1}$ \\ ${ }^{1}$ Deptartment of Pathology, Leiden University Medical Center, Leiden, The Netherlands \\ 2 Department of Surgery, Leiden University Medical Center, Leiden, The Netherlands \\ ${ }^{3}$ Department of Molecular Cell Biology, Leiden University Medical Center, Leiden, The Netherlands
}

\section{Abstract}

Aims/hypothesis. Large quantities of pure viable donor islets are necessary for clinical transplantation. At present, low yields and low viability of pancreatic islets after transplantation necessitate the use of multiple donors for a single recipient. In this study an improved method for obtaining large quantities of pure viable islets of Langerhans for transplantation was developed in the rat.

Methods. Islets of Langerhans were isolated from Albino Oxford rats. The donor pancreata were perfused in situ with iron oxide, which resulted in entrapment of iron particles in the capillaries of the islets. Subsequently, the islets were isolated by magnetic retraction. Islets obtained with this method were compared with islets obtained by density gradient-isolated islets with respect to yields, purity, and insulin production capacity. Islets isolated with the magnetic retraction method were transplanted under the renal cap- sule of streptozotocin-induced diabetic recipients. Blood-glucose levels in the recipients were monitored for 2 months after transplantation.

Results. This method yielded more pure and viable islets than the conventional protocol. No contamination of exocrine tissue was observed after isolation. Furthermore, the islets isolated by magnetic retraction stained strongly positive for insulin during the entire observation period in vitro, and produced high amounts of insulin upon a challenge with glucose. The islets that were obtained by this new protocol were suitable for safe and effective transplantation.

Conclusions/interpretation. We have shown that both the quantity and quality of islets obtained with this method were sufficient to induce insulin independence in a diabetic recipient using islets from only one donor. [Diabetologia (2004) 47:55-61]

Keywords Islets of Langerhans - pancreas - rat · isolation $\cdot$ transplantation $\cdot$ insulin $\cdot$ glucagon
Pancreas and islet transplantation are the only treatment regimens currently available to achieve a constant insulin-independent normoglycaemic state in patients with Type 1 diabetes mellitus [1,2]. Pancreas allotransplantation is surgically complex and is associated with a high morbidity $[3,4,5,6]$. For these rea-

Received: 9 May 2003 / Revised: 25 September 2003

Published online: 12 December 2003

(C) Springer-Verlag 2003

G. G. M. Pinkse ( $)$, Deptartment of Pathology, Leiden University Medical Center, Building 1, L1-Q, PO Box 9600 , 2300 RC Leiden, The Netherlands

E-mail: G.Pinkse@lumc.nl

Abbreviations: MR, magnetic retraction; DG, density gradient. sons, islet transplantation can be a feasible alternative treatment modality. Advances in immunosuppression during the last 2 years in combination with improved engraftment of islets, have now made islet transplantation a realistic therapeutic option $[2,7]$. However, human islet transplantation continues to be hindered by a limited availability of pure viable islets.

The presence of exocrine tissue after isolation can lead to complications. Such contamination can lead to autodigestion of the islets [8]. After transplantation, the contaminating exocrine tissue induces an additional inflammatory response resulting in acute damage of the islet allograft [9]. Moreover, exocrine tissue has been reported to inhibit angiogenesis and vascularization of the islet graft [10]. Furthermore, due to the low 
yields and low viability of the isolated islets, tissues from multiple donors must be used for each recipient. Therefore, development of a more rapid and effective islet purification technique is a prerequisite for further improvement of islet transplantation.

In recent years, a number of purification techniques have been applied, e.g. those using the fluorescence-activated cell sorter, or immunomagnetic beads for the removal of exocrine tissue [11, 12, 13, 14, 15, 16, 17]. Two methods to obtain purified rat islets are currently in common use. The method, i.e. handpicking of islets, is rather time-consuming. The second method utilizes a density-gradient separation during the purification step. Results with this method are inconsistent, since differences in density between acinar cells and islets are altered by conditions affected by the pancreas procurement or digestion process [18]. In addition, the use of density gradients could cause osmotic damage to purified islets. In the present study, we demonstrate how magnetic retraction $[19,20]$ can be used as a rapid and reproducible method of islet purification for transplantation. This novel technique is suitable for large-scale isolation and transplantation of viable islets of Langerhans.

\section{Methods}

Islet isolation and purification using a dextrane density gradient $(D G)$. Inbred male Albino Oxford (AO) rats $(n=20)$, weighing 200-250 g were obtained from Harlan (Horst, The Netherlands). All animals received humane care in compliance with the National Research Council's criteria for humane care as outlined in the 'Guide for the Care and Use of Laboratory Animals' prepared by the National Academy of Sciences and published by the National Institutes of Health (1985). They were anaesthetised by a subcutaneous injection containing a mixture of hypnorm and dormicum in phosphate-buffered saline (PBS). The isolation of islets and their purification with density gradients were carried out according to a previously described method [21]. The abdomen was opened, and the common bile duct was cannulated. The rat was killed, and $10 \mathrm{ml}$ of Krebs-RingerHepes (KRH) solution (25 mmol/l) containing 10\% BSA (Sigma-Aldrich Chemicals, Zwijndrecht, The Netherlands) was slowly infused into the common bile duct after clamping of the distal end just proximal to the duodenum. The pancreas was rapidly excised, and cut into small pieces $\left(2 \mathrm{~mm}^{3}\right)$ using singleedged razor blades. Islets were isolated from the surrounding exocrine tissue by enzymatic digestion using $0.7 \mathrm{mg} / \mathrm{ml}$ collagenase P (Boehringer Mannheim, Almere, The Netherlands) in $25 \mathrm{mmol} / \mathrm{l} \mathrm{KRH}$ containing $10 \% \mathrm{BSA}$ at $37^{\circ} \mathrm{C}$ for $15 \mathrm{~min}$. Purification was achieved by a discontinuous density gradient using $31 \%, 25 \%, 23 \%, 20 \%$, and $11 \%$ of Dextrane T70 (AmershamPharmacia, Roosendaal, The Netherlands), respectively. The pancreatic digest was centrifuged at $1500 \mathrm{rpm}$ for $22 \mathrm{~min}$ at $4{ }^{\circ} \mathrm{C}$. Most islets were collected from the interfaces between $23 \%$ and $25 \%$ dextrane. Islets were further purified by handpicking, using glass pipets under a stereomicroscope.

Islet isolation and purification using magnetic retraction (MR). The abdominal wall of each rat $(n=60)$ was opened by midline incision. The aorta descendus was perfused with a freshly sonicated $1.25 \%$ suspension of $\mathrm{Fe}_{3} \mathrm{O}_{4}$ (Sigma-Aldrich Chemicals, Zwijndrecht, The Netherlands) in PBS after clamping of the aorta thoracalis. To provide outflow, an incision was made in the distal vena cava. The pancreas was rapidly excised, and cut into small pieces $\left(2 \mathrm{~mm}^{3}\right)$ using single-edged razor blades. Islets were isolated from the surrounding exocrine tissue by enzymatic digestion using $0.7 \mathrm{mg} / \mathrm{ml}$ collagenase $\mathrm{P}$ in $25 \mathrm{mmol} / \mathrm{l}$ $\mathrm{KRH}$ containing $10 \% \mathrm{BSA}$ at $37^{\circ} \mathrm{C}$ for $15 \mathrm{~min}$. The digest was passed through a $210 \mu \mathrm{m}$ pore diameter metal screen. The obtained filtrate was passed through a $50 \mu$ m nylon screen (Becton Dickinson, Alphen aan de Rijn, The Netherlands) and the residue was collected. The falcon tube was placed immediately next to the magnetic pole of the Dynal Magnetical Particle Concentrator (Dynal, Oslo, Norway) for $30 \mathrm{~s}$. Successful magnetic purification was confirmed by visible accumulation of black specks on the inner sidewall of the tube next to the magnet.

Culture of islets. Islets were cultured in triplicate in RPMI 1640 (Sigma-Aldrich Chemicals, Zwijndrecht, The Netherlands) containing $100 \mu \mathrm{g} / \mathrm{ml}$ penicillin, $100 \mu \mathrm{g} / \mathrm{ml}$ streptomycin, and $10 \%$ heat-inactivated foetal calf serum in 24-well culture plates (Greiner, Alphen aan de Rijn, The Netherlands) in 95\% air $/ 5 \%$ $\mathrm{CO}_{2}$ at $37^{\circ} \mathrm{C}$. The medium was refreshed on alternate days. Islets from four rats were harvested after culture for 1, 3, and 7 days and were microscopically examined and counted using an inverted microscope (Zeiss, Sliedrecht, The Netherlands). Histological and functional analyses were carried out as detailed below.

Histological analysis of islets and pancreata. Pancreata and isolated islets from the AO rats were fixed in $4 \%$ paraformaldehyde overnight and dehydrated in a graded ethanol series. Islets were embedded in paraffin and cut in $3 \mu \mathrm{m}$ sections. Consecutive sections were immunostained for islet hormones [anti-insulin, dilution 1:200, anti-glucagon, dilution 1:700 (Dako, Glostrup, Denmark)]. All immunochemicals were diluted in PBS containing $1 \%(\mathrm{w} / \mathrm{v}) \mathrm{BSA}$. The sections were incubated with the primary antibody overnight. The sections were washed three times with PBS for $5 \mathrm{~min}$. Bound antibodies were visualized using peroxidase-labelled swine anti-rabbit IgG (Dako, Glostrup, Denmark) and developed with $\mathrm{H}_{2} \mathrm{O}_{2}$ and 3,3'-diaminebenzidinetetrahydrochloride (DAB) (SigmaAldrich Chemicals, Zwijndrecht, The Netherlands) as chromogen. The sections were counterstained with haematoxylin.

Insulin production in vitro. To evaluate the insulin release at days 1,3 , and 7 , an in vitro glucose challenge was carried out, with 20 islets per well $(n=6)$. Cultured islets were washed in RPMI-1640 and incubated in RPMI containing $2.8 \mathrm{mmol} / \mathrm{l} \mathrm{glu-}$ cose for $60 \mathrm{~min}$. This procedure was followed by an exposure to $28 \mathrm{mmol} / \mathrm{l}$ glucose for $1 \mathrm{~h}$. Finally, islets were incubated for $1 \mathrm{~h}$ at a glucose concentration of $2.8 \mathrm{mmol} / \mathrm{l}$. The supernatants of the samples were collected at the end of each incubation period. Insulin content was determined with insulin antibody radioimmunoassay kits and rat insulin standards (Linco Research, St. Charles, Mo., USA).

Insulin production in vivo after transplantation. Firstly, the possible pro-inflammatory effects of the iron particles were examined by separate injection of iron oxide $(n=2)$. For this purpose $6 \mathrm{mg} \mathrm{Fe}_{3} \mathrm{O}_{4}$ was injected under the left kidney capsule. After 1 week $(n=1)$ and 3 weeks $(n=1)$, the rats were killed and the kidneys were used for histological examination. Secondly, the capacity of magnetically isolated islets to restore normoglycaemia in diabetic recipients was evaluated in vivo. For this purpose, $17 \mathrm{AO}$ rats were made diabetic by injection of $65 \mathrm{mg} / \mathrm{kg}$ streptozotocin (Sigma-Aldrich Chemicals) via the penile vein, 5 days prior to islet transplantation.

The experimental groups were composed as follows: Group A, transplantation of 2000 islets (from four donors) per recipi- 

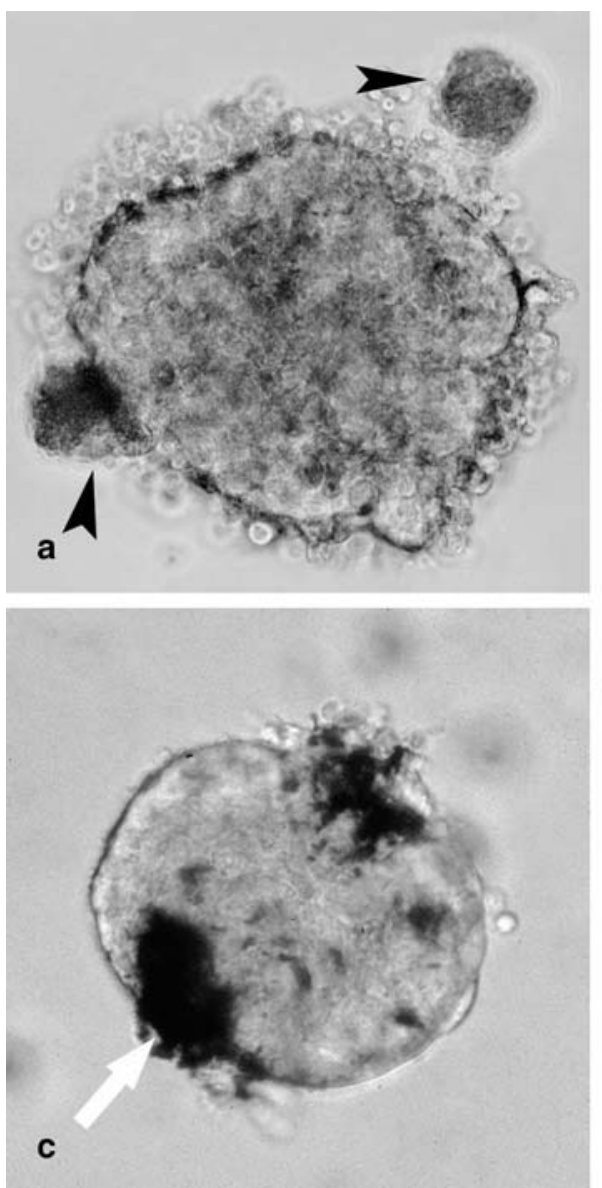

Fig. 1a-d. Culture of islets, isolated with different methods. Islet obtained with the DG method after 1 day culture (a), note the presence of attached exocrine tissue (arrowheads). Islets obtained with the MR method after 1 day (b), 3 days (c), and 7 days (d) of culture. The white arrows indicate the presence of iron oxide. Pictures were taken using inverted phase-contrast microscopy. (Original magnification $\times 200$ )

ent at the renal subcapsular site of the left kidney of diabetic AO rats $(n=9)$; Group B, transplantation of 1000 islets (from two donors) per recipient at the renal subcapsular site of the left kidney of diabetic AO rats $(n=5)$; Group C, transplantation of 500 islets (from 1 donor) per recipient at the renal subcapsular site of the left kidney of diabetic AO rats $(n=3)$.

Blood glucose was measured by the Accu-Chek Compact Analyzer (Roche Diagnostics, Almere, The Netherlands) on a daily basis until the animals were killed. After islet transplantation, the graft was removed 3 months later by nephrectomy for histological examination. In group A, two recipients were killed after 1 week and two recipients were killed after 1 month for histological examination.

Graft histology. Graft-bearing nephrectomy specimens were fixed in $4 \%$ paraformaldehyde overnight and dehydrated in a graded ethanol series. The kidneys were cut in half, embedded in paraffin, and then cut in $3 \mu \mathrm{m}$ sections. Consecutive sections were immunostained for the islet hormones insulin and glucagon.

Statistical analysis. Differences in islet yields, insulin release, and non-fasting blood glucose in the rats were compared by using the Student's $t$ test. A $p$ value of less than 0.05 was con-
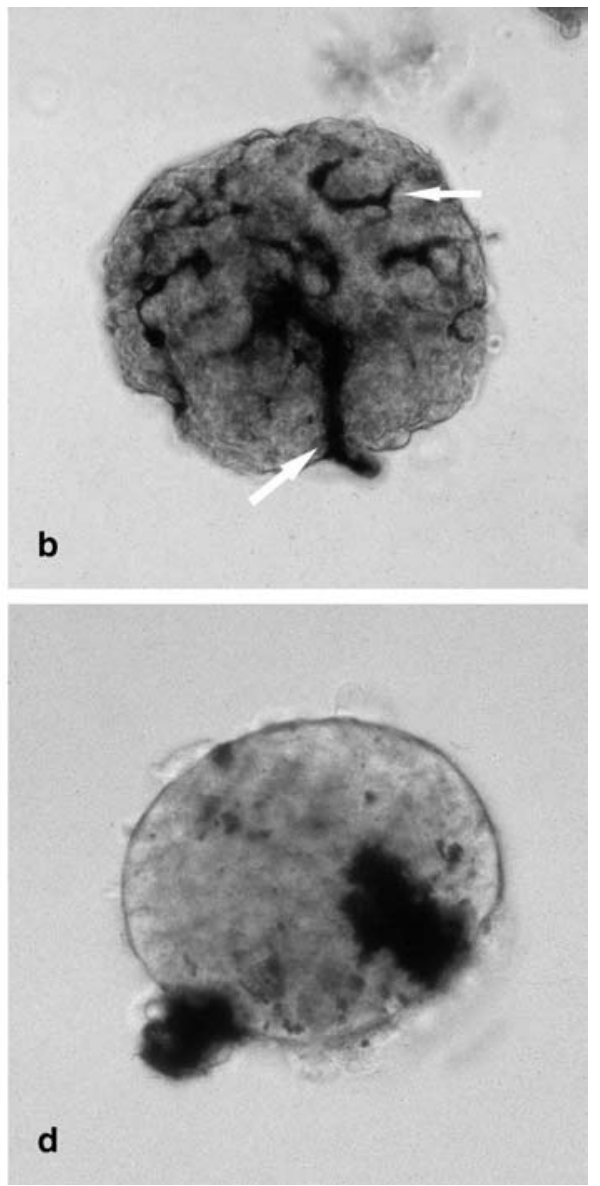

Table 1. Islet number after isolation

Isolation method Islet number per pancreas

Density Gradient (DG)

Magnetic Retraction (MR)

$312.0 \pm 19.0$

$514.6 \pm 73.6^{*}$

Numbers of islets isolated per donor pancreas by density gradient (DG) or by magnetic retraction (MR). Islets were isolated from six pancreata for each group. Data are expressed as means \pm SD. $* p<0.005$ compared to the DG group

sidered to be statistically significant. All data are expressed as means $\pm \mathrm{SD}$.

\section{Results}

Yield and purity of the isolated islets. The new magnetic isolation method was three times faster than the DG method, i.e. $1.5 \mathrm{~h}$ compared with $4.5 \mathrm{~h}$. Magnetic islet separation resulted in the isolation of highly purified, and morphologically intact islets. The MR method (Table 1) on average yielded $513 \pm 73$ islets/pancreas $(n=6)$, whereas the DG method yielded $321 \pm 19$ islets/pancreas $(n=6)(p<0.005)$ in our laboratory. Islets that had been isolated with the magnetic retraction method showed virtually no contamination of exocrine debris (Fig. 1B). By contrast, the DG method frequently resulted in exocrine tissue remaining attached to the islets (Fig. 1A). 
Islet culture. Islets were microscopically examined after 1, 3, and 7 days of culture (Fig. 1) to examine how the iron oxide had been trapped inside the islets of Langerhans. Iron particles were trapped in the microvascular network (Fig. 1b), and gradually moved

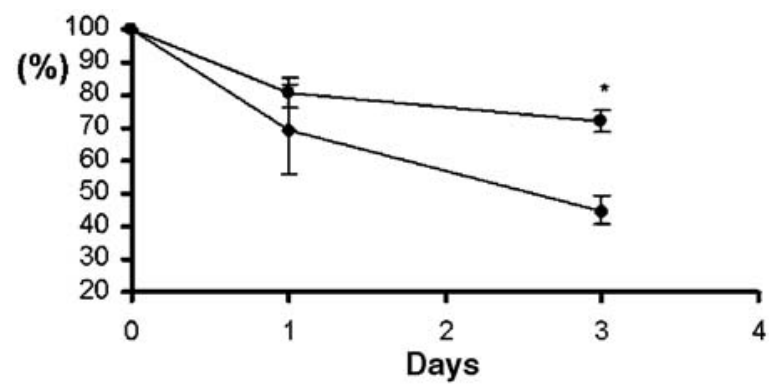

Fig. 2. Islet recovery after in vitro culture. The number of islets per well during culture was compared to the number seeded on day 0 , which was set at $100 \%$. After isolation using the MR method $(\bigcirc, n=4)$, higher percentages of islets survived during culturing compared to isolated DG islets $(\diamond, n=4)$. $* p<0.02$ compared to the number of islets during 3 days of culture after using the DG isolation method
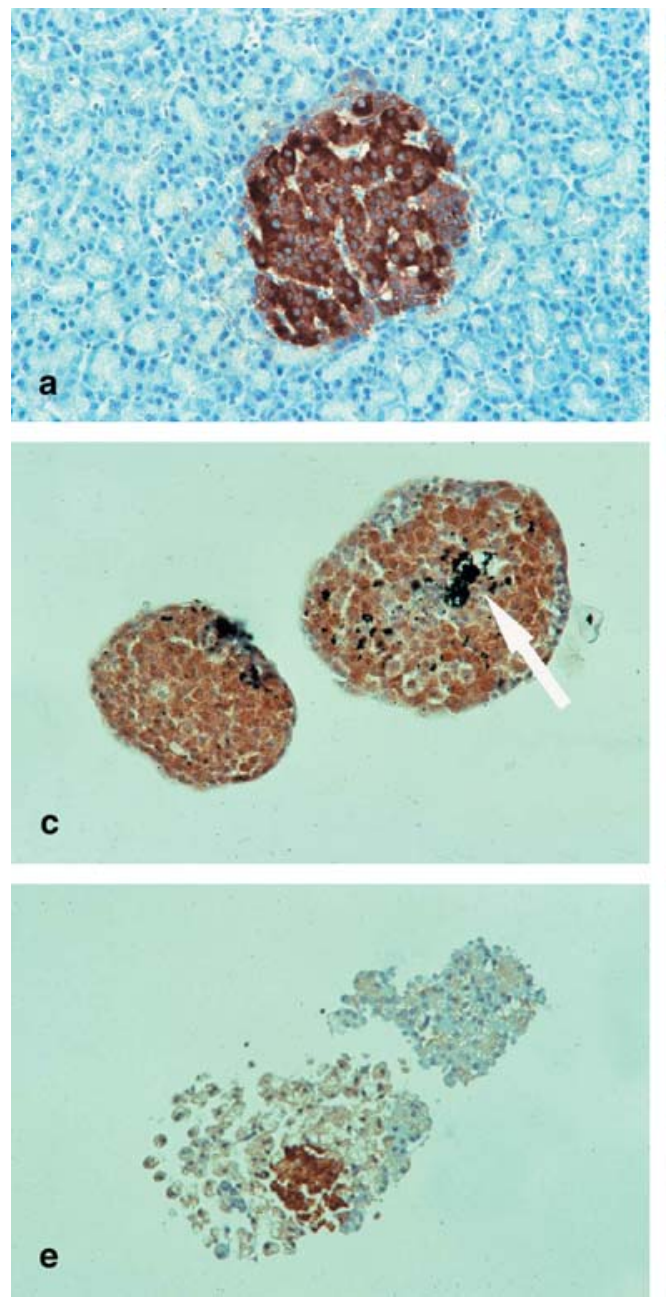

Fig. 3a-f. Islet morphology after isolation and culture. Rat pancreas sections immunostained for insulin (a), and glucagon (b). Islets isolated with the MR method stained for insulin (c) and glu- from the inner microvascular network towards the outer surface of the cultured islets during the 7 days of culturing of the islets (Fig. 1d). After 3 days of culturing, the number of islets recovered amounted to $71.8 \% \pm 3.4 \%$ in the MR group and to $44.8 \% \pm 4.3 \%$ in the DG group $(p<0.02)$ compared to the number of islets recovered on day 0 (Fig. 2).

Islet morphology. Morphologic analysis of insulinand glucagon-immunoreactive areas was done to achieve insights in the kinetics of islet morphology over time. After 7 days of culture, the islets isolated with the MR method showed the same beta cell and alpha cell composition (Fig. 3c,d) as the non-isolated islets did (Fig. 3a,b). Islets isolated with the DG method showed reduced numbers of beta cells (Fig. 3e), while alpha cells were no longer detectable after 7 days of culture (Fig. 3f).

In vitro function. To study the DG isolated and the MR-retracted islets' glucose responsiveness to insulin production, glucose challenges were carried out at
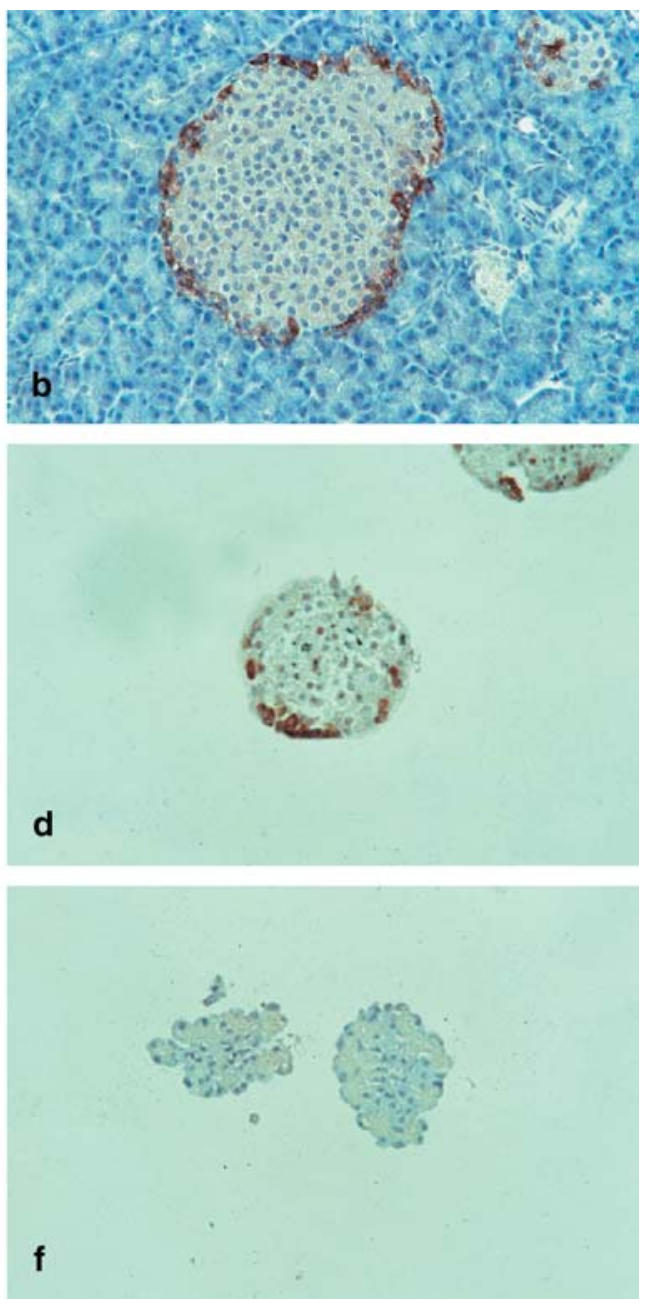

cagon (d) after 7 days of culture. Using the DG method, insulin (e) and glucagon (f) staining had disappeared in the islets after 7 days culture. The white arrow indicates the presence of iron oxide 
days 1,3 , and 7 after culture. The insulin release of the DG and the MR islets in response to $2.8,28$, and $2.8 \mathrm{mmol} / \mathrm{l}$ glucose for $1 \mathrm{~h}$ at each glucose concentration was different at day 1 and day $7(p<0.05)$ between the two groups (Fig. 4). An approximately three-fold average increase from the basal insulin secretion was observed in response to glucose stimulation $(28 \mathrm{mmol} / \mathrm{l})$, increasing from $31.8 \pm 7.5 \mathrm{pmol} /$ islet/h to $93.6 \pm 9.0 \mathrm{pmol} / \mathrm{islet} / \mathrm{h}$ for the $\mathrm{DG}$ group and from $41.5 \pm 13.3 \mathrm{pmol} / \mathrm{islet} / \mathrm{h}$ to $124.0 \pm 15.7 \mathrm{pmol} /$ islet/h for the MR islets at day $1 \quad(p<0.05)$, $35.5 \pm 11.7 \mathrm{pmol} / \mathrm{islet} / \mathrm{h}$ to $93.3 \pm 19.5 \mathrm{pmol} / \mathrm{islet} / \mathrm{h}$ for the DG group and from $29.8 \pm 8.1 \mathrm{pmol} / \mathrm{islet} / \mathrm{h}$ to $86.3 \pm 21.6 \mathrm{pmol} / \mathrm{islet} / \mathrm{h}$ for the MR group at day 3 , and $34.3 \pm 13.0 \mathrm{pmol} / \mathrm{islet} / \mathrm{h}$ to $105.4 \pm 17.1 \mathrm{pmol} / \mathrm{islet} / \mathrm{h}$ for the DG group and from $36.1 \pm 18.1 \mathrm{pmol} / \mathrm{islet} / \mathrm{h}$ to $158.3 \pm 13.3 \mathrm{pmol} / \mathrm{islet} / \mathrm{h}$ for the MR group at day 7 $(p<0.05)$. All of them returned to near basal levels after culture in low-glucose medium $(2.8 \mathrm{mmol} / \mathrm{l})$. These results indicate that the islets isolated by the iron oxide method remain viable and functionally intact after the isolation, purification and culture.

In vivo transplantation study. A pilot experiment in which $6 \mathrm{mg} \mathrm{Fe}_{3} \mathrm{O}_{4}$ was injected underneath the kidney capsule in two healthy rats triggered no morphologi-

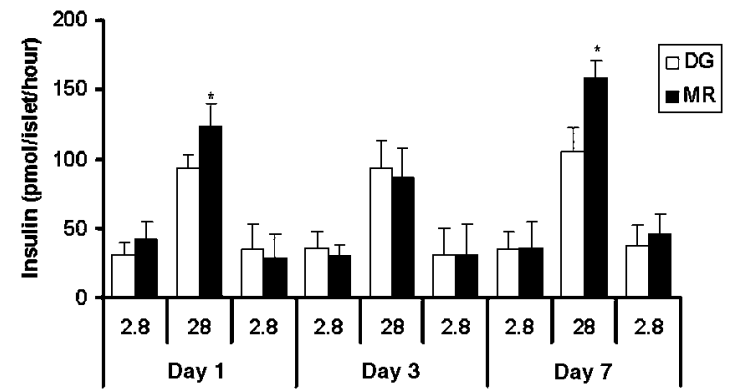

Fig. 4. Insulin secretion of DG and MR-isolated islets after glucose challenge. Glucose is given in mmol/l. Insulin release was determined of DG and MR-isolated islets in response to

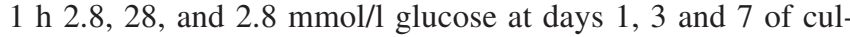
ture $(n=6)$. Data are depicted as means \pm SD. $p<0.05$ compared to the insulin secretion of islets using the DG isolation method

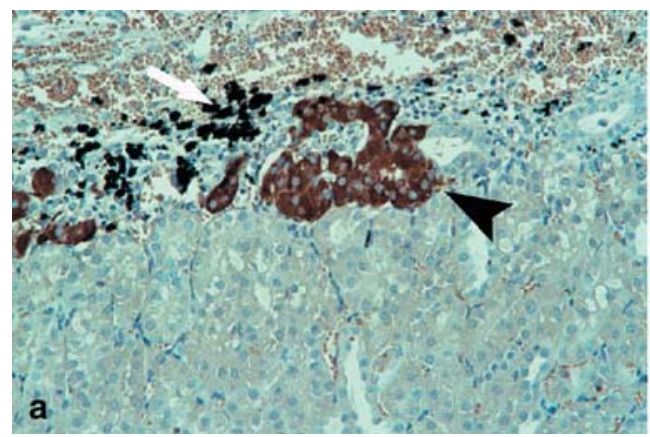

Fig. 5a, b. Morphology of islets transplanted under the kidney capsule. Magnetically retracted islets were transplanted under the kidney capsule and were stained for insulin (a) 1 week and cally detectable immune response after 7 days and after 21 days. Subsequently, it was investigated whether the islets isolated with MR had the ability to restore normoglycaemia in streptozotocin-induced diabetic rats. The rats received either 2000, 1000 or 500 islets and were respectively called group A, B and C. They all corrected the diabetic state within 1 to 2 days after engraftment. Intact islets, detected by the method of insulin staining, were still present beneath the renal capsule at 1 week and at 1 month after transplantation (Fig. 5a,b). Stable graft function was maintained for at least 2 months, until graft-bearing nephrectomy reinduced hyperglycaemia in all recipients within $48 \mathrm{~h}$ (Fig. 6), which indicated that normoglycaemia was not the result of recovery from the beta cells after the streptozotocin treatment.

\section{Discussion}

The treatment of patients with Type 1 diabetes mellitus has focused on both preventing and retarding the development of chronic complications. Whole-pancre-

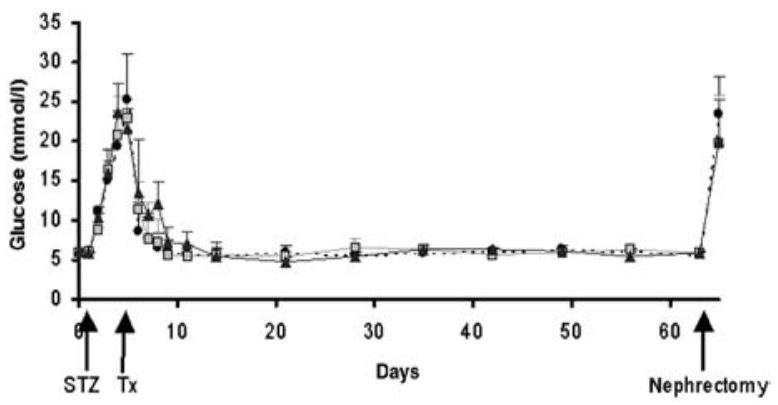

Fig. 6. Serum glucose (mean values \pm SD) in diabetic recipient rats after transplantation of islets with different donor:recipient ratios. At day 1, rats were made diabetic by injection of streptozotocin (STZ). MR-isolated islets (Tx) were placed beneath the renal capsule of syngenic AO diabetic rats. Black circles show recipients of 2000 islets (group A) $(n=5)$. Grey squares show recipients of 1000 islets (group B) $(n=5)$ and the black triangles show recipients of 500 (group C) $(n=3)$. Recurrence of hyperglycaemia is seen within 2 days after removal of the graft by nephrectomy on day 62

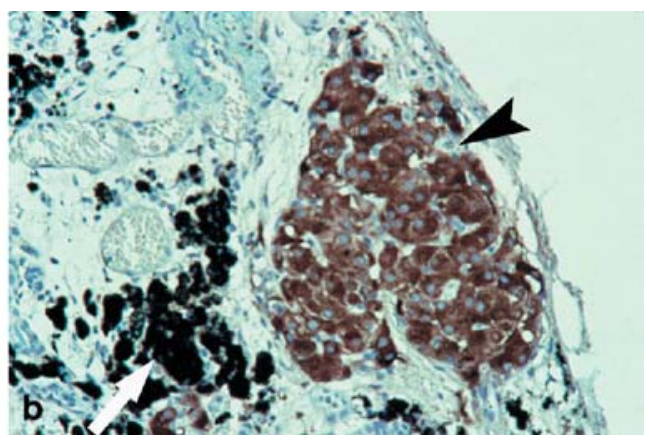

(b) 1 month after transplantation. Inflammatory cells were not detected. The white arrows indicate the presence of the iron oxide. The black arrowheads indicate the islets of Langerhans 
as allotransplantation can restore metabolic control and prevent fluctuations of blood glucose, which are responsible for severe long-term complications. Unfortunately, whole-pancreas allotransplantation has many limitations and disadvantages, such as significant morbidity and mortality [22]. Transplantation of isolated islets as a free cellular graft is a less invasive alternative. Advances in immunosuppression during the last 2 years in combination with improved graft quality have made islet transplantation a realistic therapeutic option [2]. In the successful series of islet transplantations by the Edmonton group, metabolic correction required islet preparations from two to four pancreata that were procured under optimized conditions [2]. A single donor transplant of islets is ideal but not realistic because of the limited availability of high-quality donor pancreata. Moreover, material is lost during the islet isolation procedure due to the techniques currently applied. Therefore, the islet isolation procedure must be further improved in order to obtain a higher number of viable islets from available organs.

In this study, a novel and rapid method of islet purification is presented. We developed an improved method for obtaining higher yields of islets of Langerhans from the pancreas by adapting a method used for isolating glomeruli from murine kidneys [19, 20]. Since islets of Langerhans have a similar angioarchitecture to mouse glomeruli, the most plausible explanation for the trapping of iron oxide particles $(70 \mathrm{~nm})$ is that they form aggregates, which have a tendency to accumulate selectively in the smallest capillaries (5.27 micrometers) [23]. After collagenase digestion, the islets of Langerhans can be isolated with the help of magnetic retraction.

With this novel method both the yield and the level of purity of the islets obtained is higher than with the classical method used at our laboratory. This new method has the advantage of eliminating the need for density gradient and handpicking of the islets, and, therefore, is less time consuming. This method also avoids prolonged contact of the islets with the proteolytic enzymes released by the exocrine tissue. In addition, the elimination of a discontinuous density gradient prevents osmotic damage of the islets. Until presently, the challenge has been to produce solutions that minimize acinar tissue swelling without compromising islet yield and viability [8].

Contamination by exocrine tissue has been reported as being detrimental for the process of angiogenesis and vascularization of transplanted islets [10]. After isolation with the iron oxide magnetic retraction method, the islet preparations had virtually no contamination of exocrine debris and showed no remaining exocrine tissue attached to the islets. This could be of great importance for optimizing human islet isolation. The yield of islets obtained with the MR method was two times higher than the yield of islets obtained with the DG method. The islet isolation was carried out three times faster using MR than by using current isolation methods. Since apoptosis is a time-related mechanism, a reduction in the time required for the isolation procedure might influence islet survival [24]. We observed that the iron particles had gradually moved from the inner microvascular network towards the outer surface of the cultured islets during the 7 days that the islets were cultured.

The recovery after $48 \mathrm{~h}$ of culture was approximately $76 \%$ with the new method. This is in sharp contrast with another study, which reports a recovery of $56 \%$ after $48 \mathrm{~h}$ of islet culture and disintegration of damaged islets in culture [8]. This indicates that our new isolation method gives a higher quality of islets. After 7 days of culture, both the cellular composition and the glucose-induced insulin production per islet had not decreased. In contrast, other studies using cultured islets show a gradual decline in insulin content $[25,26]$, and the glucagon-producing cells have often disappeared. It has been shown that peripheral glucagon secreting cells (alpha cells) are lost during most of the isolation procedures. The glucose-induced insulin release depends not only on the insulin-containing beta cells, but also on their interactions with other types of endocrine cells within the islet [27]. Therefore, a change in the islet cells' composition could affect insulin release. After isogeneic transplantation of the islets, in vivo function was adequate for rapid reversal of the diabetic state within 1 day in all recipients. This resulted in stable graft function for more than 2 months. Furthermore, our study shows that the presence of iron oxide in the islets under the renal capsule does not lead to inflammatory reactions, and would thus appear not to be harmful to the recipient. The iron oxide crystals were never seen within the cells, but always remained trapped in the small capillaries. The subsequent virtual expulsion of the iron particles after either culture, or transplantation could be the result of neoangiogenesis generated by recovering endothelium, as has recently been shown to occur in cultured islets [28]. Both the in vitro insulin secretion and the in vivo insulin production after islet transplantation are excellent. We found that iron oxide had no detrimental effect on the islets and their beta-cell function. However, long-term harmful effects of magnetic iron oxide ( $>4$ months) cannot be excluded from our study.

This new islet isolation procedure provides a highly reliable method for obtaining improved islet yields suitable for transplantation. In human situations, most recipients still need islet preparations isolated from more than one donor to provide a sufficient islet mass for achieving insulin independence [2, 29]. With this study we intended to show that, with this new method, both quality and quantity of the islets obtained from one donor are sufficiently high for maintaining one diabetic recipient's insulin-independence. This new 
method can also be expected to improve the results of human islet transplantation, because it seems to have the ability of overcoming some of the obstacles successful clinical transplantation is currently facing.

Acknowledgements. The authors wish to thank D. Hoogervorst, J. Rijkelijkhuizen, B. van der Zouwen and H. Baelde for excellent technical assistance. The authors thank Dr. M. Eikmans and Dr. B. Roep for critical reading the manuscript. Part of this study was carried out with financial support of BTS grant 98161 from Senter, Ministry of Economic Affairs, The Netherlands.

\section{References}

1. Bretzel RG, Brandhorst D, Brandhorst H et al. (1999) Improved survival of intraportal pancreatic islet cell allografts in patients with type-1 diabetes mellitus by refined peritransplant management. J Mol Med 77:140-143

2. Shapiro AM, Lakey JR, Ryan EA et al. (2000) Islet transplantation in seven patients with type 1 diabetes mellitus using a glucocorticoid-free immunosuppressive regimen. N Engl J Med 343:230-238

3. Manske CL (1999) Risks and benefits of kidney and pancreas transplantation for diabetic patients. Diabetes Care 22 Suppl 2:B114-B120

4. Smets YF, Westendorp RG, Pijl JW van der et al. (1999) Effect of simultaneous pancreas-kidney transplantation on mortality of patients with type-1 diabetes mellitus and endstage renal failure. Lancet 353:1915-1919

5. Sutherland DE, Gruessner RW, Dunn DL et al. (2001) Lessons learned from more than 1000 pancreas transplants at a single institution. Ann Surg 233:463-501

6. Oberholzer J, Triponez F, Mage R et al. (2000) Human islet transplantation: lessons from 13 autologous and 13 allogeneic transplantations. Transplantation 69:11151123

7. Roep BO, Stobbe I, Duinkerken G et al. (1999) Auto- and alloimmune reactivity to human islet allografts transplanted into type 1 diabetic patients. Diabetes 48:484-490

8. London NJ, Swift SM, Clayton HA (1998) Isolation, culture and functional evaluation of islets of Langerhans. Diabetes Metab 24:200-207

9. Gotoh M, Maki T, Satomi S, Porter J, Monaco AP (1986) Immunological characteristics of purified pancreatic islet grafts. Transplantation 42:387-390

10. Heuser M, Wolf B, Vollmar B, Menger MD (2000) Exocrine contamination of isolated islets of Langerhans deteriorates the process of revascularization after free transplantation. Transplantation 69:756-761

11. Ricordi C, Lacy PE, Scharp DW (1989) Automated islet isolation from human pancreas. Diabetes 38 Suppl $1: 140-142$
12. Korbutt GS, Pipeleers DG (1993) Rat pancreas preparation for cold storage and subsequent islet cell isolation. Transplantation 56:500-503

13. Davies JE, James RF, London NJ, Robertson GS (1995) Optimization of the magnetic field used for immunomagnetic islet purification. Transplantation 59:767-771

14. Jindal RM (1995) The use of zinc binding dyes and fluorescence-activated islet sorters. Pancreas 11:316-318

15. Gray DW, Gohde W, Carter N, Heiden T, Morris PJ (1989) Separation of pancreatic islets by fluorescence-activated sorting. Diabetes 38 [Suppl 1]:133-135

16. Davies JE, Winoto-Morbach S, Ulrichs K, James RF, Robertson GS (1996) A comparison of the use of two immunomagnetic microspheres for secondary purification of pancreatic islets. Transplantation 62:1301-1306

17. Fujioka T, Terasaki PI, Heintz R et al. (1990) Rapid purification of islets using magnetic microspheres coated with anti-acinar cell monoclonal antibodies. Transplantation 49:404-407

18. White SA, James RF, Swift SM, Kimber RM, Nicholson ML (2001) Human islet cell transplantation-future prospects. Diabet Med 18:78-103

19. Gauthier VJ, Mannik M (1988) A method for isolation of mouse glomeruli for quantitation of immune deposits. Kidney Int 33:897-899

20. Baelde JJ, Bergijk EC, Hoedemaeker PJ, Heer E de, Bruijn JA (1994) Optimal method for RNA extraction from mouse glomeruli. Nephrol Dial Transplant 9:304-308

21. De Vos P, Van Straaten JF, Nieuwenhuizen AG et al. (1999) Why do microencapsulated islet grafts fail in the absence of fibrotic overgrowth? Diabetes 48:1381-1388

22. Robertson RP, Davis C, Larsen J, Stratta R, Sutherland DE (2000) Pancreas and islet transplantation for patients with diabetes. Diabetes Care 23:112-116

23. Henderson JR, Moss MC (1985) A morphometric study of the endocrine and exocrine capillaries of the pancreas. Q J Exp Physiol 70:347-356

24. Frisch SM, Francis H (1994) Disruption of epithelial cell-matrix interactions induces apoptosis. J Cell Biol 124:619-626

25. Wang R, Li J, Rosenberg L (2001) Factors mediating the transdifferentiation of islets of Langerhans to duct epithelial-like structures. J Endocrinol 171:309-318

26. Wang RN, Rosenberg L (1999) Maintenance of beta-cell function and survival following islet isolation requires reestablishment of the islet-matrix relationship. J Endocrinol 163:181-190

27. Bertuzzi F, Berra C, Socci C et al. (1995) Glucagon improves insulin secretion from pig islets in vitro. J Endocrinol 147:87-93

28. Linn T, Schneider K, Hammes HP et al. (2003) Angiogenic capacity of endothelial cells in islets of Langerhans. FASEB J 17:881-883

29. Toso C, Oberholzer J, Ris F et al. (2002) Factors affecting human islet of Langerhans isolation yields. Transplant Proc 34:826-827 